\title{
Looking back and looking forward
}

\author{
With 12 issues under our collective editorial belt, we look back and reflect on the journal's first year of life.
}

$\mathrm{N}$ ature Human Behaviour launched last January with the aim of publishing research of outstanding significance from across the entire spectrum of disciplines that research topics relevant to individual or collective human behaviour. Over the past year, we published 81 research Letters and Articles that collectively span the social, biological, health and physical sciences, ranging from psychology, economics, political science, sociology and anthropology to neuroscience, genetics and evolution; to epidemiology, public health and behavioural medicine; to statistical physics.

At launch, we made a point to specifically encourage the submission of interdisciplinary and transdisciplinary research, which doesn't find an easy home in disciplinary journals (see also our December 2017 Editorial). Over the year, we have published several manuscripts that straddle different disciplines. For instance, Gomez-Lievano and colleagues combined insights from economics and cultural evolution to build a theory of urban scaling. Power applied network science methods to ethnographic data from rural South India, finding that religiosity is associated with greater prosociality. A team of anthropologists and psychologists used a neuroarchaeological approach to identify the brain networks that may have underlain Early Stone Age toolmaking.

From the outset, we also committed to publishing research with potential impacts beyond academia. For example, Caspi et al. provided evidence that supports social policies that target very young children at risk of adverse adult outcomes. Castilla-Rho and colleagues showed that how likely people in different regions are to comply with regulations for groundwater management depends on each region's cultural, socioeconomic, institutional and physical conditions. In countries with individualistic cultures, people are more likely to be vaccinated when they know more about herd immunity, found Betsch and colleagues, suggesting that vaccine advocacy should utilize prosocial nudges to close immunity gaps in those countries. Using the 2014 New York Police Department slowdown as a natural

Table 1 | Top-scoring magazine articles - altmetric (lifetime scores as of
19 December 2017)
\begin{tabular}{ll} 
Altmetric score & Article title \\
\hline 3,214 & Why people prefer unequal societies \\
2,475 & A manifesto for reproducible science \\
1,193 & Viewing addiction as a brain disease promotes social injustice \\
1,087 & Moral outrage in the digital age \\
837 & Should social science be more solution-oriented?
\end{tabular}

experiment, Sullivan and O'Keeffe showed that reductions in proactive policing can reduce major crime.

We are strongly supportive of efforts to strengthen the robustness and reproducibility of research, and are keenly aware of the role scientific journals need to play in this domain. Our first issue set the tone in two ways: we published 'A manifesto for reproducible science', a Perspective written by a group of leading voices advocating a series of measures for the improvement of the transparency and reproducibility of scientific research; and we announced our adoption of registered reports as a key format encapsulating the principles of robust, reproducible science. With registered reports, research projects are granted acceptance in principle before the data have been collected, if the editors and reviewers determine the research question to be important and of broad relevance, and the study design and analysis plan to be robust and sufficiently powered. This format neutralizes several biases harmful for science, including publication bias, HARK-ing (hypothesizing after the results are known) and $P$-hacking. At the time of writing, we have accepted in principle two stage 1 registered reports, with more under consideration. We are hoping to receive many more submissions in the coming year and encourage any interested researchers unfamiliar with the format to contact us to discuss how they could adopt it in their research.

In addition to our research content, we published more than 160 magazine articles - Reviews, Perspectives, Comments, World Views, News \& Views, Research Highlights and more. These pieces reflect the entire scope of the journal, offer the opportunity for discussion and debate, put forward novel and occasionally provocative ideas, and address pressing societal challenges: poverty, migration, health, well-being, inequality, discrimination, climate change, sustainability. They also represent a broad spectrum of voices, including contributors beyond academia. Take a look at our five most discussed magazine articles over the past year in Table 1 .

We feel strongly about engaging with the research communities we serve in person as much as possible. This past year, the Nature Human Behaviour editors attended more than 40 conferences, workshops and laboratory/site visits. We are looking forward to meeting and talking to many more of you in 2018 .

We are deeply grateful to all scientists who entrust their work to us - regardless of outcome, we value the opportunity to consider your work and are respectful of the painstaking effort that went into each paper submitted to us. We thank our more than 900 unique reviewers, who invested significant time and effort to evaluate our submissions in 2017 and helped strengthen them for publication in Nature Human Behaviour or elsewhere. Our advisory board members have provided us with unique insights into their fields of research and key developments, supporting our efforts. And, thank you, our readers, for engaging with the journal and making 2017 a thrilling year for us.

Here's to a new year of exciting science! $\square$

Published online: 8 January 2018

https://doi.org/10.1038/s41562-017-0287-y 